\title{
Challenges in Dissolution Testing
}

\author{
Margareth R. C. Marques*, William Brown, Gabriel \\ Giancaspro, Natalia Davydova, Edith Chang, Jeanne Fringer, \\ Walter Hauck, and Anthony DeStefano \\ U. S. Pharmacopeia, Rockville, MD, USA
}

e-mail:mrm@usp.org

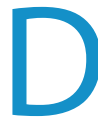
issolution testing of dosage forms is an activity in continuous development, both for improving equipment and its performance qualification and for selecting the best test conditions. The U. S. Pharmacopeia (USP) held a workshop coordinated by some USP expert panels at its headquarters in Rockville, MD, on June 11 and 12,2012, to present and discuss the ongoing projects related to dissolution. This paper presents a summary of the discussions held at this meeting.

\section{PERFORMANCE TESTS FOR SEMISOLID DRUG PRODUCTS}

The evaluation of drug release for semisolid dosage forms such as ointments, gels, creams, and lotions is an important tool during product development and for post-approval changes (1). A new USP General Chapter $<1724>$ Semisolid Drug Products-Performance Tests was developed to describe the equipment that could be used for this evaluation and to provide instructions on how to run the tests and how to use it for comparison of post-approval changes. This new chapter was published for the first time in Pharmacopeial Forum (PF) 37(5). (PF is available free of charge at www.usppf.com.) The comments received after this publication were evaluated and incorporated into the text. The revised version of this chapter was published in PF 38(3). This chapter contains an introduction to the theory behind the in vitro performance tests for semisolid dosage forms, a description of the tests, and instructions on using the available equipment (vertical diffusion cell, immersion cell, and a cell that can be used with USP Apparatus 4) and evaluating the results to assess sameness of the drug product after postapproval changes. The comments received after publication in PF 38(3) include:

- Avoid any correlation with in vivo data.

- Emphasize that this test is applicable only for product development and post-approval changes and has no application as a quality control tool.

- Specify the temperature range for the vertical diffusion cell as $\pm 1^{\circ} \mathrm{C}$.

- Update some of the equipment drawings.

- Make small changes on the instructions on how to set up some of the equipment.

- Correct the formula for amount released.

${ }^{*}$ Corresponding author.
The discussions during the workshop addressed the high variability associated with drug release results. Recommendations were made to include in the general chapter a section on equipment troubleshooting, method validation, and membrane selection, and to do some collaborative studies to improve the instructions on how to run the test. As a result of the discussions, a new USP expert panel will be created to address the revision and expansion of this general chapter. More information on the activities related to this topic can be obtained from Margareth R. Marques, Ph.D., at MRM@ usp.org.

\section{LIQUID-FILLED CAPSULES}

The dissolution testing of liquid-filled capsules may require some conditions or approaches not covered by USP General Chapter $<711>$ Dissolution. A new USP General Chapter <1094> Liquid-filled Capsules-Dissolution Testing and Related Quality Attributes was developed to overcome these deficiencies. This new chapter was published in PF 38(1), and it addresses manufacturing and packaging issues that can affect dissolution testing (e.g., cross-linking in gelatin capsules). Also, it contains some recommendations on how to develop and validate a dissolution procedure for liquid-filled capsules. During the discussions at the workshop, it was recommended to expand the chapter to include all types of capsules, not just liquid-filled. The biggest challenges are how to demonstrate the presence of cross-linking in gelatin capsules and how to use enzymes when the dissolution medium contains surfactant. Depending on the type of filling, USP Apparatus 3 may be more appropriate because it provides a more turbulent environment. More information on the activities related to this topic can be obtained from Margareth R. Marques, Ph.D., at MRM@usp.org.

\section{DISSOLUTION OF DIETARY SUPPLEMENTS}

USP General Chapter <2040> Disintegration and Dissolution of Dietary Supplements contains recommendations on the apparatus and procedure to evaluate the release of the active ingredient from capsules and tablets containing dietary supplements. In the case of vitamin-mineral dosage forms, the chapter recommends the dissolution test for one index vitamin and folic acid, if present. At the workshop, a dietary supplement company presented data showing that the current 
conditions recommended in the chapter are not appropriate for liquid-filled capsules containing folic acid. They conducted in vivo bioavailability studies to demonstrate that folic acid was released in vivo. They have ongoing projects to improve the test as written in USP, and they will report the results.

General Chapter <2040> was revised to include a new specification for the beakers used in the disintegration test, disintegration testing of delayed-release soft shell capsules, and dissolution testing of vitamin A tablets and lipid-filled soft shell gelatin capsules. More information can be obtained from Natalia Davydova, Ph.D., at ND@ usp.org.

Most USP monographs for botanical dietary supplements do not require a dissolution test but may require rupture or disintegration tests. Many factors affect dissolution of phytochemicals including water solubility, stability in the Gl tract, and so forth. Poor dissolution of dosage forms containing botanicals may explain the lack of demonstration of clinical effect of some products. As new delivery mechanisms are developed and dietary supplements are made more bioavailable, unanticipated effects (e.g., toxicity due to drug interaction) may be manifest.

\section{USE OF ENZYMES IN THE DISSOLUTION TESTING OF GELATIN CAPSULES}

USP General Chapter $<711>$ Dissolution allows the use of enzymes when gelatin capsules and gelatin-coated tablets fail the dissolution test and states the conditions for the addition of pepsin or pancreatin to the dissolution medium. The meaning of failing the test is not very clear, and pepsin does not show optimal activity in all the $\mathrm{pH}$ ranges stated in the chapter. In addition, there are no instructions on the procedure to be adopted in the case when the dissolution medium contains surfactant. A USP expert panel was created to address all these issues, and a paper will be published in $P F$ explaining all the changes that will be made in Chapter $<711>$ Dissolution and the rational for these changes. A new activity assay method for pepsin was proposed in PF 38(3). This new procedure is essentially that same procedure that will be adopted by the Food Chemicals Codex. The influence on the pepsin activity assay by the presence of surfactants is being evaluated. More information on the activities related to this topic can be obtained from Margareth R. Marques, Ph.D., at MRM@usp.org.

\section{PERFORMANCE VERIFICATION TEST (PVT)}

USP holds that both the mechanical calibration and performance verification tests (PVT) are necessary to evaluate both USP Apparatus 1 and 2, and neither procedure is sufficient alone. Recent changes to the PVT require demonstration of consistency of results with a range. The use of salicylic acid tablets and chlorpheniramine extended-release tablets to verify the performance of USP Apparatus 2 and USP Apparatus 3 , respectively, has been removed from USP General Chapter $<711>$ Dissolution. More information on the activities related to this topic can be obtained from William Brown at WEB@usp.org.

The new PVT criteria include limits on the geometric mean (two-sided) and \%CV (one-sided). A single-stage or doublestage approach is chosen by the lab and should be specified in the laboratory procedures. A calculation tool is available at http://www.usp.org/usp-nf/compendial-tools/pvt-calculatorterms-use- 0 . The acceptance criteria are based on a large collaborative study. The wider specifications reflect the interlaboratory variability found in the study.

USP General Chapter <1058> Analytical Instrument Qualification describes the procedures and documentation appropriate for qualification of analytical equipment. The PVT is used periodically to show that the dissolution apparatus is performing as expected. Mechanical calibration can be used as a complementary or substituted approach as described by USP, FDA, and ASTM. Dissolution laboratories must decide whether to use PVT, mechanical calibration, or both to control significant sources of variability per FDA requirements. The qualification of USP Apparatus 3 no longer requires the use of Chlorpheniramine Extended-Release Tablets. Without a PVT, detailed mechanical verification is critical. The mechanical qualification of USP Apparatus 4 includes verification of vessel horizontality and dimensions, temperature, and flow control. No internal vibration should occur. Handling of PVT failures when all mechanical measurements are in specification requires full evaluation of each PVT parameter. Insufficient degassing, bad bearings, insufficiently clean vessels, and so forth, can cause failures. This emphasizes the importance of the PVT.

The USP Dosage Form Performance Laboratory is working on the development of a new lot of Prednisone Tablets with less sensitivity to vessel irregularity and deaeration and an increased shelf life. Through a collaborative study, USP and FDA labs are evaluating the effects of vibration on USP Apparatus 2 to establish limits for vibration. Analyses of USP Apparatus 3 and Apparatus 4 are being done to determine the critical parameters affecting hydrodynamics and the potential for using salicylic acid tablets for the PVT for Apparatus 4. More information on the activities related to this topic can be obtained from Erika Stippler, Ph.D., at ESS@usp.org.

\section{DEVELOPMENT AND VALIDATION OF DISSOLUTION PROCEDURES}

USP General Chapter <1092> The Dissolution Procedure: Development and Validation is being redesigned by a subcommittee of the USP General Chapters-Dosage Forms Expert Committee. The chapter will be expanded to include more information on filter selection and compatibility; determination of the effects of media, $\mathrm{pH}$, and so 
forth, on drug solubility; choosing a suitable dissolution medium and apparatus; sample handling; and the quantitative steps. A section on automation will be added, and the validation section of this chapter will be expanded. More information on the activities related to this topic can be obtained from William Brown at WEB@usp.org.

\section{ALTERNATIVES FOR DISSOLUTION TESTING- RUPTURE VERSUS DISINTEGRATION TEST}

The circumstances where a dissolution test may be replaced by a disintegration or a rupture test were discussed. The solubility of the drug substance and the characteristics of the capsule filling are extremely important and should be taken into consideration. A representative of FDA explained that the agency has not yet fully accepted the rupture test as an alternative for disintegration or dissolution testing. If a proposal is made with scientific justification, FDA might consider it.

\section{REFERENCES}

1. Nonsterile Semisolid Dosage Forms_Scale-Up and Postapproval Changes: Chemistry, Manufacturing, and Controls; In Vitro Release Testing and In Vivo Bioequivalence Documentation; Guidance for Industry; U.S. Department of Health and Human Services, Food and Drug Administration, Center for Drug Evaluation and Research (CDER), U.S. Government Printing Office: Washington, DC, 1997. http://www.fda.gov/downloads/ Drugs/GuidanceComplianceRegulatorylnformation/ Guidances/UCM070930.pdf (accessed July 9, 2012). 